\title{
Dual-smoothing for Marine Oil Spill Segmentation
}

\author{
Mengmeng $\mathrm{Di}^{1}$, Huajun Song ${ }^{1}$, Chunbo $\mathrm{Luo}^{2}$, Christos Grecos ${ }^{3}$, and Peng Ren ${ }^{1}$
}

\begin{abstract}
We present a novel marine oil spill segmentation method that characterizes two smoothing modules at the label level and the pixel level separately. At the label level, we exploit the rolling guidance filter for smoothing the label cost volumes. It enables scale-aware labelling and thus alleviates the ambiguous segmentation that blurs the detailed structures of oil spills. At the pixel level, we adapt a cooperative model for smoothing higher order pixel variations, which has the potential of preserving elongated strips that often arise in oil spills. We integrate the two smoothing modules operating at different levels into an energy minimization formulation, which is referred to as dual-smoothing. The coupling of the two smoothing modules enables an effective complement to each other such that the specific structures of oil spills are accurately characterized. We compute the optimal labelling of the dual-smoothing framework based on graph-cuts. The proposed dual-smoothing framework is especially effective in segmenting elongated and detailed oil spills, and the experiment results demonstrate its advantages over threasholding and graphcuts based segmentations.
\end{abstract}

Index Terms-Oil spill segmentation, Dual-smoothing, Graphcuts.

\section{INTRODUCTION}

O IL spill disasters and accidents have occurred frequently in the past decades and caused serious damages to the affected natural environment and ecosystem [1]. It is thus particularly helpful if oil spills can be detected at an early stage. To monitor and detect oil spills through remote sensing technologies has also been an important research topic [2]. Of all the popular monitoring platforms, satellite based Synthetic Aperture Radar (SAR) has played an important role due to its capacity of all-weather and all-time operation [3][4]. The key to explore the full potential of SAR for oil spill detection is to effectively process the obtained images, where image segmentation is particularly important [5]. The challenges and difficulties in accurate SAR segmentation come from the facts that oil spills often exhibit irregular patterns and are exposed to excessive noise. Elongated strips of oil spill areas are one class of common shapes in SAR images that are difficult to preserve in segmentation. Furthermore, the boundaries of oil spill areas exhibit large variations at different scales, which may easily cause ambiguities in segmentation.

Though oil spill segmentation has been studied for decades, the above challenges have not been fully addressed yet in literature - existing methods tend to apply generic image segmentation strategies to the oil spill segmentation scenario but ignore the intrinsic features of oil slicks [6]. For instance, thresholding is a type of generic segmentation methods and is a widely used in segmenting SAR images into oil areas and nonoil areas [7]. This pixel-wise strategy is efficient but extremely sensitive to noise. One solution to address this disadvantage is to perform smoothing on the image for denoising and then threshold the pixel gray values. However, the smoothing filters tend to reduce the useful detailed structures and noise together and lead to labelling ambiguity. A more general solution to improve the robustness of pixel-wise methods is to incorporate pairwise terms as the denoising procedure and formulate an integrated objective function for both thresholding and smoothing. Discrete optimization methods such as graph-cuts are then used to compute the segmentation. These graphcuts strategies are not only effective in segmenting generic images but also achieve state-of-the-art performance in oil spill segmentation [8]. However, the pairwise terms in graphcuts encourage consistent segmentations by penalizing the assignment of different labels to neighboring pixels. It can cause oil strip areas to be cut off or erased in segmentation.

To overcome the drawbacks of the existing methods and address the challenges in oil spill segmentation, we present a dual-smoothing framework for accurately segmenting the oil spills from SAR images. We formulate the oil spill segmentation as a labelling problem. Our smoothing scheme operates at the label level and pixel level separately. The label level smoothing elaborates boundary segmentation at different scales and the pixel level smoothing favors the shape of long oil strips. We finally optimize the dual-smoothing framework based on graph-cuts and obtain the segmentation results.

Polarimetric SAR data [9] have also been broadly exploited for oil spill detection. In this regard, one notable recent study in literature is the Bayesian oil spill segmentation approach [10], which achieves state of the art performance. On the other hand, we investigate oil spill segmentation mainly form an image processing point of view and do not consider the polarimetric information. Therefore, our framework and the polarization based method may possibly complement each other in various imagery situations.

\section{Preliminaries}

For an image with $N$ pixels, let $I=\left\{I_{1}, I_{2}, \ldots, I_{N}\right\}$ denote the set of the pixel gray values, with $I_{i}$ representing the gray value of the pixel $i$. We formulate the oil spill segmentation as a labelling problem. Let $X=\left\{x_{1}, x_{2}, \ldots, x_{N}\right\}$ denote the binary variable set where $x_{i}$ is the label assigned to the pixel $i$. The segmentation is to assign a label $x_{i} \in\{0,1\}$ to every pixel $i$ of an image. In the oil spill segmentation scenario, if one pixel is assigned the label 0 , it is segmented to the oil area, and if one pixel is assigned the label 1, it is segmented to the non-oil area. We aim to compute the optimal labelling $X$ which gives the segmentation results. The labelling problem is normally formulated in terms of minimizing an energy function consisting of unary terms and pairwise terms, where unary terms penalize the inconsistence for assigning a label to 
a pixel and pairwise terms penalize the assignment of different labels to adjacent pixels. Our contribution is to describe how to develop new unary terms and pairwise terms which result in an accurate oil spill segmentation.

\section{LABEL LEVEL SMOOTHING}

In this section we describe how to perform smoothing on label cost volumes, which results in a robust unary term. One cost volume measures the inconsistence between a pixel and a label. One traditional definition of the cost volume $\tilde{E}_{i}\left(x_{i}\right)$ for assigning label $x_{i} \in\{0,1\}$ to the pixel $i$ is formulated as

$$
\left\{\begin{array}{l}
\tilde{E}_{i}(0)=-\log P\left(I_{i} \mid B\right) \\
\tilde{E}_{i}(1)=-\log P\left(I_{i} \mid O\right)
\end{array}\right.
$$

where $O$ and $B$ indicate oil area and non-oil background area, respectively. In our work, the probabilities $P\left(I_{i} \mid B\right)$ and $P\left(I_{i} \mid O\right)$ are formulated by Gaussian mixture models (GMMs) and estimated through Expect-Maximization (EM) algorithms. The features related to wind speeds, incident angles or oil types are thus encoded by the GMM model parameters. For labelling a pixel $i$, one traditional thresholding method is to compare the two cost volumes $\tilde{E}_{i}(0)$ and $\tilde{E}_{i}(1)$ for it. If $\tilde{E}_{i}(0) \leq \tilde{E}_{i}(1)$, the pixel $i$ is labelled 0 , and otherwise it is labelled 1. The thresholding strategy is straightforward but very sensitive to noise. To neutralize the noise influence, the thresholding can be done after pre-smoothing the noisy image. However, the smoothing on pixels is low-passing filtering in nature. It blur the high-frequency features such as the boundaries and details of the oil spill area such that labelling ambiguity might arise. In contrast to processing the image at pixel level, we apply smoothing at the label level. Specifically, we exploit a rolling guided filter to smooth the cost volumes for both labels 0 and 1 over the whole image pixels. The detailed operations are described as follows

$E_{i}\left(x_{i}\right)=\frac{\sum_{j:(i, j) \in \mathcal{A}} \exp \left(-\frac{d_{i j}^{2}}{2 \sigma_{s}^{2}}-\frac{\left\|\tilde{E}_{i}\left(x_{i}\right)-\tilde{E}_{j}\left(x_{j}\right)\right\|^{2}}{2 \sigma_{r}^{2}}\right) \tilde{E}_{i}\left(x_{i}\right)}{\sum_{j:(i, j) \in \mathcal{A}} \exp \left(-\frac{d_{i j}^{2}}{2 \sigma_{s}^{2}}-\frac{\left\|\tilde{E}_{i}\left(x_{i}\right)-\tilde{E}_{j}\left(x_{j}\right)\right\|^{2}}{2 \sigma_{r}^{2}}\right)}$

where $\mathcal{A}=\{(i, j) \mid$ the pixels $i$ and $j$ are spatially adjacent $\}$, $d_{i j}$ is the spatial distance between the pixels $i$ and $j$ in the image. $\sigma_{s}$ and $\sigma_{r}$ denote the standard deviations, controlling the spatial and range weights, respectively. The new unary term $E_{i}\left(x_{i}\right)$ is more desirable than (1) in penalizing pixel-label inconsistence. The reason for its effectiveness is two-fold. First, rather than processing pixel gray values, the smoothing in (2) operates on different cost volumes for different labels separately. Cost volumes for different labels are contrastive such that they effectively complement each other in preserving oil spill structures in smoothing. Second, the rolling guided filter itself is edge and scale aware [11], favoring the preservation of boundaries and details of oil spills at different scales.

Fig. 1 illustrates some processing results for one small patch of a SAR image containing oil spills. Fig. 1(a)-1(d) illustrate the cost volumes before and after being smoothed by the rolling guided filter. The original cost volumes for both label 0 and 1 are rather noisy, while the smoothed cost volumes are comparatively clean. Furthermore, the smoothed cost volumes contrastively preserve labelling structures. The oil area in the middle of the patch is not clearly characterized in the cost volume for label 1 (i.e. background) in Fig. 1(d). In contrast, it presents dominant saliency in the cost volume for label 0 (i.e. oil spill area) in Fig. 1(b). The two smoothed cost volumes complement each other such that the labelling ambiguity caused by smoothing can be partially neutralized.

\section{Pixel LeVel SMoothing}

In this section, we describe how to develop a pixel level smoothing term which favors elongated oil strip segmentation. The processing are based on pairs of adjacent pixels. For a pair of adjacent pixels $(i, j) \in \mathcal{A}$, we define the pairwise term $E_{i j}\left(x_{i}, x_{j}\right)$ as follows

$$
E_{i j}\left(x_{i}, x_{j}\right)=\left|x_{i}-x_{j}\right| \cdot s_{i j}
$$

where $s_{i j}=\exp \left(\frac{-\left|I_{i}-I_{j}\right|^{2}}{2 \sigma^{2}}\right)$.

The pairwise term (3) penalizes the different labelling between a pair of adjacent pixels. Therefore, the minimization of (3), sometimes along with a unary term such as that introduced in Section III, tends to lead to an over-smoothing effect such that oil strips might be partially erased in the segmentation. To overcome the disadvantages of the straightforward smoothing in (3), we exploit a cooperative model to establish the smoothing term at the pixels level. This is commenced by grouping the set of adjacent pixel pairs $\mathcal{A}$ into $K$ clusters by performing $K$-means on the similarity measure $s_{i j}$. The obtained $K$ classes of pairs of adjacent pixels are denoted as $\mathcal{A}_{1} \cdots \mathcal{A}_{K}$. In this scenario, pixel pairs across the boundary of a strip are very likely to form clusters because of their close similarities. Here the individual elements in one subset $\mathcal{A}_{k}$ are pixel pairs, not individual pixels. To render a more convenient representation for characterizing individual pixels in $\mathcal{A}_{k}$ rather than pixel pairs, we use the notation $\mathcal{P}_{k}$ for denoting the set of pixels involved in $\mathcal{A}_{k}$. The set $\mathcal{P}_{k}$ is a subset of the image pixel set. Let $X_{1} \cdots X_{K}$ denote the label sets for pixels in the subsets $\mathcal{P}_{1} \cdots \mathcal{P}_{K}$, respectively. Specifically, one binary variable in $X_{k}$ is the label for one individual pixel in $\mathcal{P}_{K}$. A higher-order term $E_{\mathcal{P}_{k}}\left(X_{k}\right)$, which incorporates all pairwise terms associated with $\mathcal{A}_{k}$, is defined over the set $\mathcal{P}_{k}$ of pixels

$$
E_{\mathcal{P}_{k}}\left(X_{k}\right)=\min \left\{\sum_{(i, j) \in \mathcal{A}_{k}} E_{i j}\left(x_{i}, x_{j}\right), T\right\}
$$

where $T$ is a thresholding parameter. The higher-order term (4) can be thought of being a cooperative model because the pairwise terms involved in $\mathcal{A}_{k}$ cooperate with each other in terms of certain operations within the model. In our work, we use sum and thresholding operation introduced in [12] as the cooperative model. Other cooperative functions can also be applied subject to monotonically increasing concavity.

The higher-order term (4) favors the preservation of oil elongated strips in segmentation because it alleviates the penalty for assigning different labels to adjacent pixel pairs within a cluster. Even if pixels in a cluster formed by cross boundary pixel pairs are differently labelled, the minimum of (4) is 


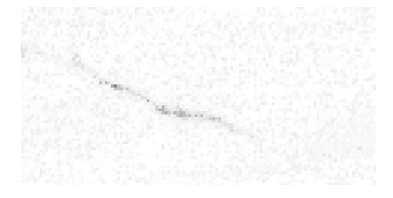

(a) Oil cost before filtering.

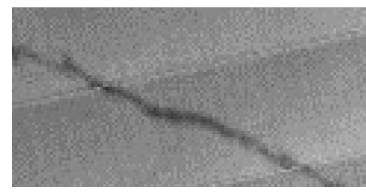

(e) Original image.

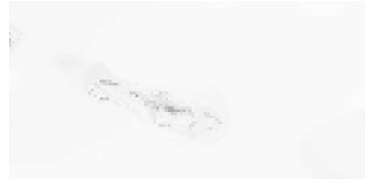

(b) Oil cost after filtering.

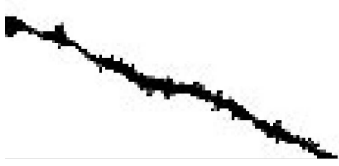

(f) Manual segmentation.

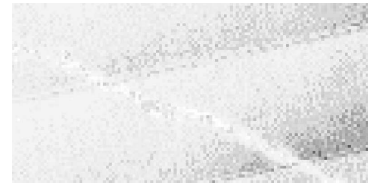

(c) Background cost before filtering.

(d) Background cost after filtering.

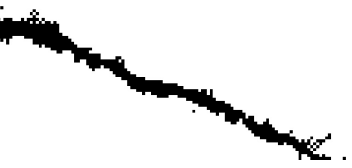

(h) Dual-smoothing.

Fig. 1: Processing results for a small patch of one SAR image containing oil spills.

not greater than the threshold $T$. Therefore, the minimization of (4) allows different labels across strip boundary and thus encourages the preservation of elongated strips of oil spills.

\section{DuAl-SMOOThing FOR SEgmentation}

The label smoothing presented in Section [II and pixel smoothing presented in Section IV] operate at different level separately. In order to take the advantages of both schemes, we integrate them into a dual-smoothing framework in terms of energy minimization. Specifically, we integrate the unary terms (2) and the higher-order terms (4) into an energy function

$$
E(X)=\sum_{i=1}^{N} E_{i}\left(x_{i}\right)+\sum_{\mathcal{P}_{k}} E_{\mathcal{P}_{k}}\left(X_{k}\right)
$$

Different configurations for $X=\left[x_{1}, x_{2}, \cdots, x_{N}\right]$ represent different segmentation results. The minimization of (5) optimizes the penalties encoded by both the label smoothing and the pixel smoothing and thus shares the merits from these two levels. However, the energy function (5) contains higher-order terms, i.e. $E_{\mathcal{P}_{k}}(\cdot)$, and cannot be minimized by using popular discrete optimization methods such as graph-cuts and belief propagation, which are just applicable for pairwise cases. To render an efficient computation, the higher-order term $E_{\mathcal{P}_{k}}$ (4) is reformulated as a quadratic pseudo-boolean function (QPBF) [12][13] as follows

$$
\begin{array}{r}
E_{\mathcal{P}_{k}}\left(X_{k}\right)=T+\min _{h_{g}, y_{i j}}\left\{\sum _ { ( i , j ) \in \mathcal { A } _ { k } } \beta _ { i , j } \left(\left(x_{i}+x_{j}-2 y_{i j}\right) h_{g}\right.\right. \\
\left.\left.-2\left(x_{i}+x_{j}\right) y_{i j}+4 y_{i j}\right)-T h_{g}\right\}
\end{array}
$$

where $y_{i j}$ and $h_{g}$ are auxiliary binary variables. After the QPBF transformation, the higher-order term $E_{\mathcal{P}_{k}}(\cdot)$ is transformed into pairwise terms, i.e. there is no product of variables in (6) with order greater than pairwise. Substituting (6) into (4), we have the energy function (4) formulated by the linear combination of unary terms and pairwise terms. We use the min-cut/max-flow algorithm [14] to compute the minimization of the energy function and obtain an optimal labelling configuration, resulting in the final oil spill segmentation.
Fig. 1(g) and Fig. 1(h) illustrate the segmentation results of graph-cuts and dual-smoothing which are obtained through employing (3) and (4), respectively ${ }^{1}$. Compared with the manual segmentation illustrated in Fig. 1(f), the oil strip in Fig. 1(g) is partially erased, which is caused by the oversmoothing effect of the pairwise term (3). In contrast, the oil strip is better preserved in Fig. 1(h), which is benefited from the advantage of the higher-order term (4) in characterizing elongated boundaries of oil strips.

\section{EXPERIMENTS}

In this section, we experimentally compare the performance of the proposed dual-smoothing framework with that of the thresholding method [7] and the graph-cuts method [8] in oil spill segmentation. We use SAR images with VV polarization obtained from NOWPAP database ${ }^{2}$ in our experiments. The data are 32-bit floating point based calibrated images. The experiments are implemented by using Matlab 2013b with an embedded $\mathrm{C}++$ compiler.

Fig. 2 illustrates the experimental results for oil spills shaped in long strips or even more complicated patterns. Specifically, Fig. 2(a) is the original image of oil spill; Fig. 2(b) gives the manual segmentation of the oil spill areas; Fig. 2(c) demonstrates the thresholding segmentation result; Fig. 2(d) shows the segmentation result of graph-cuts; and Fig. 2(e) is the segmentation result of the proposed dual-smoothing method. Compared with the manual segmentation of oil spill, the threshold segmentation result has excessive noise and poor accuracy. The dual-smoothing method shows superior performance over the traditional thresholding and graph-cuts methods in preserving elongated stripes and detailed structures - a little elongated stripes which is neglected in Fig. 2(d) has been successfully identified and shown in Fig. 2(e). Fig. 2(f) illustrates the receiver operating characteristic curves(ROC) of the three methods, from which we can see that the ROC curve

\footnotetext{
${ }^{1}$ The segmentation results in Fig. 1(g) and Fig. 1(h) are both obtained by incorporating the unary terms 10 and 2 , respectively. The integration of the unary term and higher-order term is introduced in Section $\mathrm{V}$

${ }^{2} \mathrm{http} / / /$ cearac.poi.dvo.ru/en/db/
} 


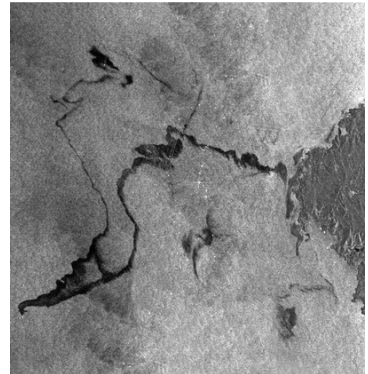

(a) Original image.

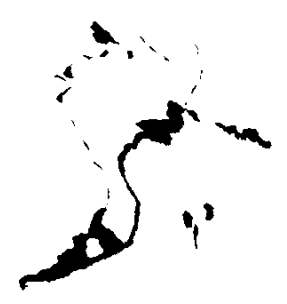

(d) Graph-cuts.

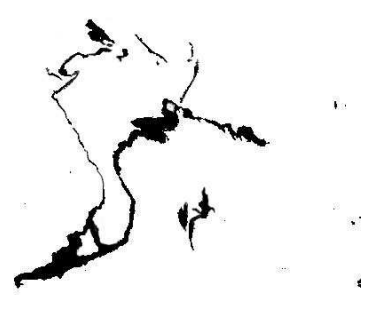

(b) Manual segmentation.

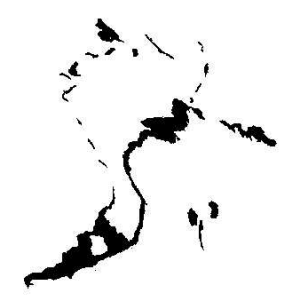

(e) Dual-smoothing.

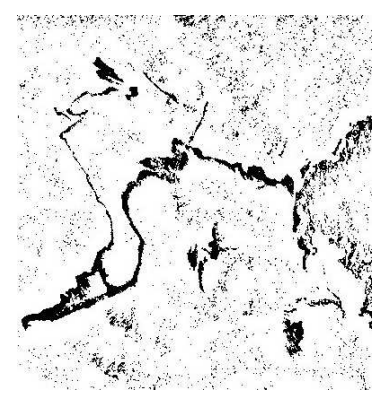

(c) Threshold segmentation.

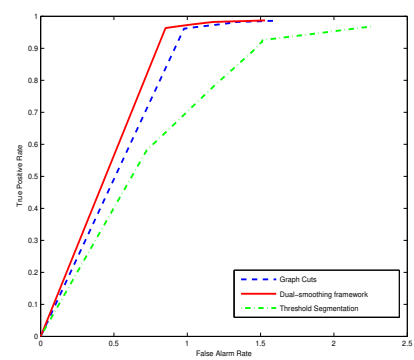

(f) ROC curves.

Fig. 2: The segmentation results for one whole SAR image.

of the dual-smoothing framework is much closer to the manual segmentation.

Fig. 3 shows the experiment results from a SAR image with an extremely slim oil stripe under random noise. Fig. 3(a) is the original image; Fig. 3(b) gives the manual segmentation of oil spill; Figs. 3(c), 3(d) and 3(e) show the results of thresholding, graph-cuts and dual-smoothing, respectively. It is clear that the dual-smoothing method outperforms alternative methods, as evaluated by the ROC curve in Fig. 3(f) where it has the steepest ROC curve among the three methods.

Fig. 4 demonstrates the experiment results for processing a SAR image under wave disturbances, which are shaped in a clear order and not easy to filter out as random noise. Here we observe that though the dual-smoothing framework in this orderly disturbed situation does not appear to be as effective as those in Figs. 2 and 3, it still shows advantage over the two alternative segmentation methods, especially when the false alarm rate is low, as shown in Fig. 4(f).

Table 1 gives the segmentation accuracies and precisions for alternative methods over two hundred patches containing elongated strips extracted from the satellite SAR images. We can see that the dual-smoothing framework outperforms the other two method in both accuracy ( $\frac{\# \text { correctly segmented pixel }}{\# \text { all pixels }}$ ) and precision ( $\frac{\# \text { correctly segmented oil pixels }}{\# \text { oil pixels segmented }}$ ) along with their standard deviations.

\section{CONCLUSIONS AND FUTURE WORK}

This paper presents a dual-smoothing framework for segmenting oil spill areas in SAR images. Theoretical and em-
TABLE I: Accuracy/Precesion/Robustness

\begin{tabular}{c|c|c|c}
\hline \hline Method & Thresholding & Graph-cuts & Dual-smoothing \\
\hline Accuracy & $0.6406 \pm 0.1353$ & $0.7623 \pm 0.1686$ & $0.8070 \pm 0.0819$ \\
\hline Precision & $0.7847 \pm 0.1829$ & $0.7856 \pm 0.0940$ & $0.8033 \pm 0.0893$ \\
\hline \hline
\end{tabular}

pirical studies reveal that our method is especially effective in segmenting detailed and elongated strips of oil spills. Additionally, we leave a free parameter $T$ in the higher order term for tuning. Our study is conducted from an image processing point of view, and one possible direction for our future work is to investigate how to involve polarization characterization, which would effectively complement the visual information. Furthermore, based on the combination of polarization and visual information, we will also address more challenging problems such as the discrimination between oil spill areas and low wind areas, ship wakes or biogenic slicks for developing a more general and robust segmentation framework.

\section{REFERENCES}

[1] Y. Loya, and B. Rinkevich. Effects of oil pollution on coral reef communities. Marine Ecology Progress Series, 3(16): 180, 1980.

[2] A. B. Salberg, O. Rudjord, Anne H. Schistad Solberg. Oil Spill Detection in Hybrid-Polarimetric SAR Images. IEEE Transactions on geoscience and remote sensing, 10(52): 6521-6523, 2014

[3] The first Marine oil spill emergency response plan. China Ocean News, 905(1), 2000 .

[4] F. Del Frate, A. Petrocchi, and J. Lichtenegger. Neural networks for oil spill detection using ERS-SAR data. IEEE Transactions on Geoscience and Remote Sensing, 38(5): 2282-2287, 2000. 


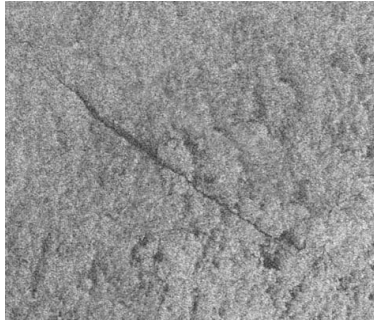

(a) Original image.

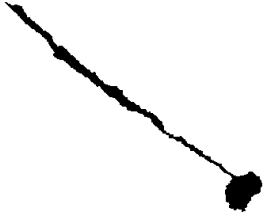

(d) Graph-cuts.

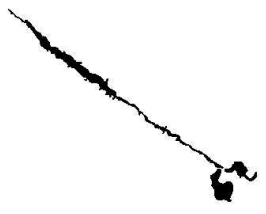

(b) Manual segmentation.

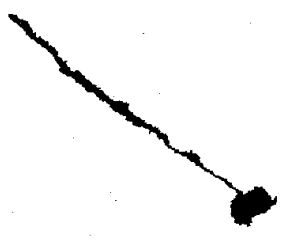

(e) Dual-smoothing.

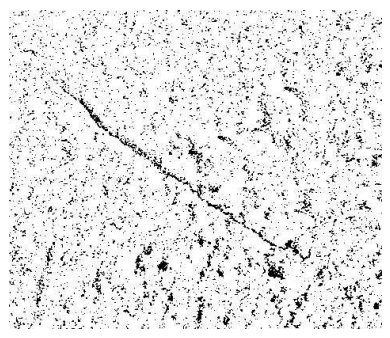

(c) Threshold segmentation.

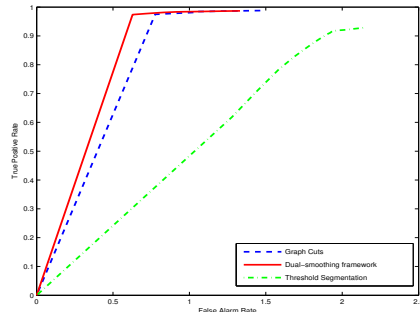

(f) ROC curves.

Fig. 3: The segmentation results for a SAR image patch under random noise.

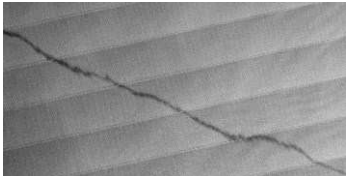

(a) Original image.

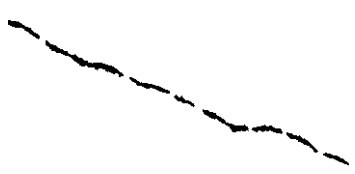

(d) Graph-cuts.

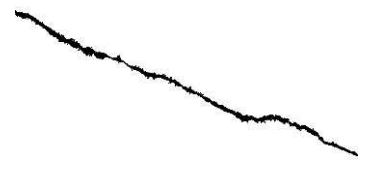

(b) Manual segmentation.

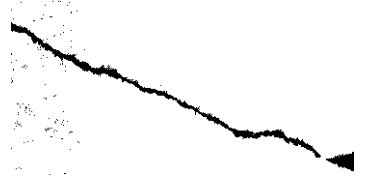

(e) Dual-smoothing

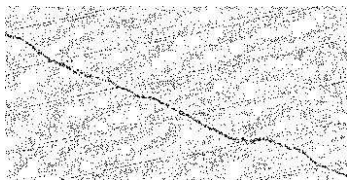

(c) Threshold segmentation.

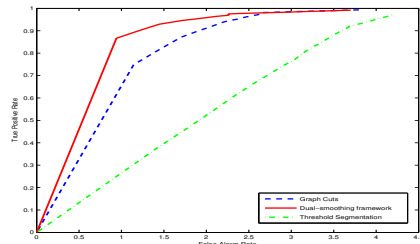

(f) ROC curves.

Fig. 4: The segmentation results for a SAR image patch under wave disturbances.

[5] M. Marghany, and M. Hashim. Texture entropy algorithm for automatic detection of oil spill from RADARSAT-1 SAR data. International Journal of the Physical Sciences 5(9): 1475-1480, 2010.

[6] A. Solberg, C. Brekke, and P. Husoy. Oil spill detection in radarsat and envisat SAR images. IEEE Transactions on Geoscience and Remote Sensing, 45(3): 746-755, 2007.

[7] A. Sheta, M. Alkasassbeh, and M. Braik. Detection of oil spills in SAR images using threshold segmentation algorithms. International Journal of Computer Applications, 2012.

[8] S. Pelizzari, and J. Bioucas-Dias. Oil spill segmentation of SAR images via graph cuts. IEEE Geoscience and Remote Sensing Society, 2007.

[9] F. Bandiera and G. Ricci, Slicks detection on the sea surface based upon polarimetric SAR data. IEEE Geoscience Remote Sensing Letters, 3(2): 342346, 2005.

[10] F. Bandiera, A. Masciullo, G. Ricci, A Bayesian approach to Oil Slicks Edge Detection based on SAR data. IEEE Transactions on Geoscience and Remote Sensing, 5(52): 2901-2909, 2014.

[11] Q. Zhang, X. Shen, L. Xu, and J. Jia. Rolling Guidance Filter. European Conference on Computer Vision, 2014.

[12] P. Kohli, A. Osokin, and S. Jegelka. A principled deep random field model for image segmentation. IEEE Conference on Computer Vision and Pattern Recognition, 2013.

[13] S. Jegelka, and J. Bilmes. Submodularity beyond submodular energies: coupling edges in graph cuts. IEEE Conference on Computer Vision and Pattern Recognition, 2011.

[14] Y. Boykov, and V. Kolmogorov. An experimental comparison of Mincut/Max-flow algorithms for energy minimization in vision.IEEE Transactions on Pattern Analysis and Machine Intelligence, 26(9): 1124-1137, 2004. 